\title{
Substituent-Dependent Chemoselective Synthesis of Highly Functionalized Benzo[h]quinolines and 4-Benzylpyrans from 2-Methyl-5-nitro-benzonitrile
}

\author{
Rahul Panwar \\ Shally \\ Ranjay Shaw \\ Amr Elagamy \\ Chandan Shah \\ Ramendra Pratap*
}

Department of Chemistry, University of Delhi, North campus,

Delhi, India-110007, India

ramendrapratap@gmail.com

rpratap@chemistry.du.ac.in

Dedicated to Prof. Uli Kazmaier on his $59^{\text {th }}$ birthday
Received: 01.10.2018

Accepted after revision: 04.10.2018

Published online: 30.10 .2018

DOI: 10.1055/s-0037-1610665; Art ID: so-2018-d0051-op

License terms: CC

Abstract A facile, efficient and atom-economic synthesis of highly substituted benzo[ $h]$ qninolines was established by reaction of 2-methyl-5-nitrobenzonitrile with suitably functionalized $2 \mathrm{H}$-pyran-2-ones under basic conditions. We observed that the presence of a thiomethyl group at the C-4 position of pyran provides 6-aryl-4-(2-cyano-4-nitrobenzyl)-2-oxo-2H-pyran-3-carbonitrile exclusively without any trace of benzo[h]quinolines. Depending on the nature of the functional group at C-4 of the pyran ring, different products were achieved. To probe the mechanism, we performed control experiments and isolated 3-(1-amino-7-nitro-3-thiophen-2-yl-naphthalen-2-yl)-3-piperidin-1-yl-acrylonitrile, which, on further treatment with base, provided the benzo[h]quinolines. The structure of one the products was characterized by singlecrystal X-ray diffraction.

Key words $2 \mathrm{H}$-pyran-2-one, 2-methyl-5-nitro-benzonitrile, benzo[h]quinoline, 4-benzylpyran, chemoselective

Polycyclic $\mathrm{N}$ - and $\mathrm{O}$-heterocycles such as amsacrine, benzo[c]phenanthridines, ellipticines, intoplicine and coralyne exhibit excellent biological properties such as DNA topoisomerase inhibition and anticancer activity. ${ }^{1-5}$ Among the $\mathrm{N}$-heterocycles, fused and isolated quinolines are very important and exhibit a wide range of biological properties including anaesthetic, ${ }^{6 \mathrm{a}}$ antiestrogenic, ${ }^{6 \mathrm{~b}-\mathrm{c}}$ antimalarial, ${ }^{7}$ anti-HIV, ${ }^{8}$ anticancer, ${ }^{9}$ antitubercular, ${ }^{10}$ and antimicrobial activities. ${ }^{11}$ They have also been widely used in agrochemical areas ${ }^{12}$ and in material chemistry. ${ }^{13}$ Recently, our research group has reported the synthesis and anticancer activity of functionalized benzo[ $h$ ]quinolines. ${ }^{14}$ This class of compounds has also been used in the construction of nano and meso structures that exhibit novel electronic and photonic properties. ${ }^{15}$

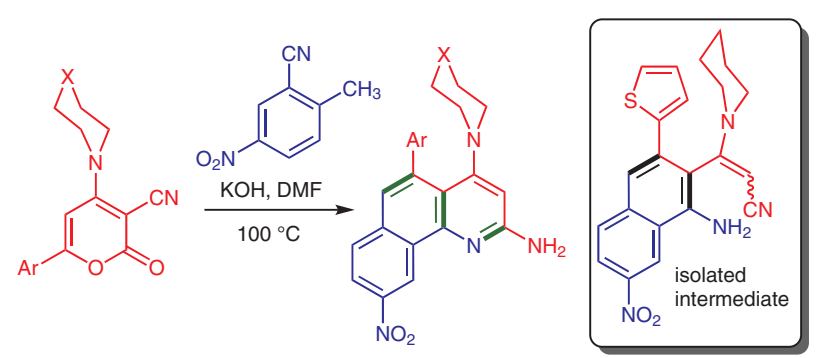

Various synthetic methodologies have been reported for the synthesis of fused and isolated quinolines, from Skraup, Doebner and von Miller syntheses, ${ }^{16-19}$ through Diels-Alder reactions 20 and Friedlander condensations reactions. ${ }^{21}$ Quinolines have also been synthesized by palladium, ${ }^{22}$ copper, ${ }^{23}$ nickel, ${ }^{24}$ and zinc ${ }^{25}$ catalyzed inter- and intramolecular cyclization reactions. The reaction of aryl isothiocyanates, alkynes, and alkyl triflates also provides quinolines and fused quinolines. ${ }^{26}$ In another approach, partially reduced benzo[ $h]$ quinolines were obtained by reaction of arylidenes and 1-tetralone in the presence of ammonium acetate and sodium methoxide. ${ }^{27}$ Benzo[ $\left.h\right]$ quinolines have also been afforded by reaction of 6-methoxy-1-tetralone and methyl propiolate in saturated ammonical methanol. ${ }^{28}$ Ram and co-workers reported the synthesis of partially reduced benzo[ $h$ ]quinolines by reaction of 5,6-dihydro-2oxobenzo[ $h]$ chromenes with formamidine or benzamidine and S-methylisothiourea under basic conditions. ${ }^{29}$ Recently, our group established a one-pot chemoselective synthesis of benzo[ $h]$ quinolines by reaction of 2-pyranones and 2-cyanomethylbenzonitrile in DMF in the presence of sodamide. This reaction requires extended reaction times (up to 36$50 \mathrm{~h}$ ) with conventional heating but 55 minutes under microwave irradiation. ${ }^{30-32}$ In this connection, we wanted to use 2-methyl-5-nitrobenzonitrile as the carbanion source instead of 2-cyanomethylbenzonitrile to study its effect on reactivity with 2-pyranone and to achieve the benzo $[h]$ quinolines without a nitrile group, because regioselective removal of a nitrile group is difficult and requires additional steps.

To initiate our studies, the precursor 6-aryl-4-methylsulfanyl-2-oxo- $2 \mathrm{H}$-pyran-3-carbonitriles 4 were synthesized by reaction of 2-cyano-3,3-bismethylthioacrylate and a range of aryl/heteroaryl methyl ketones in dimethylsulfoxide under basic conditions at room temperature ${ }^{33 a-c}$ and 
amination of 6-aryl-4-methylthio-2-oxo-2H-pyran-3-carbonitriles with secondary amines in ethanol provided 6aryl-4-amino-2-oxo-2H-pyran-3-carbonitriles 1 . $^{34 \mathrm{a}-\mathrm{e}}$

To study the optimization, 6-(4-chlorophenyl)-2-oxo-4(piperidin-1-yl)-2H-pyran-3-carbonitrile (1) and 2-methyl5-nitrobenzonitrile (2) were selected as a model substrates. Initially, we performed the reaction using triethylamine as base in dimethyl sulfoxide (DMSO) and DMF at $100{ }^{\circ} \mathrm{C}$, but no reaction was observed (Table 1, entries 1 and 2). Similarly, the use of sodamide in DMF or DMSO at either room temperature or higher temperatures led mainly to starting material being recovered (entries 3-6); while use of sodium hydride afforded complex mixtures (entries 7-9) containing starting materials and desired product, along with unidentified decomposition products. When the reaction was performed in DMF using $\mathrm{KOH}$ as base at room temperature for $12 \mathrm{~h}, 45 \%$ yield of the desired product was isolated (entry 10) and when the same reaction mixture was heated at $100{ }^{\circ} \mathrm{C}$ for $6 \mathrm{~h}, 93 \%$ of the desired benzo[ $h$ ]quinoline was isolated (entry 11). Use of DMSO instead of DMF, lowered the yield of product (entry 12). Use of potassium tert-butoxide as a base in DMF at $100{ }^{\circ} \mathrm{C}$ provided the desired product in $65 \%$ yield (entry 13 ); whereas using cesium carbonate as base was did not afford the desired product (entry 14). By using lithium hydroxide and sodium hydroxide under the same conditions noted in entry 11 , a lower yield and complex reaction mixture were observed, respectively (entries 15 and 16). Use of $\mathrm{KOH}$ in water gave no reaction and starting materials were recovered (entry 17) and using $\mathrm{KOH}$ in DMSO for 6 hours provided only $40 \%$ yield of the desired product (entry 18). Thus, we chose the reaction of 2pyranone and 2-methyl-5-nitrobenzonitrile with potassium hydroxide in DMF at $100{ }^{\circ} \mathrm{C}$ as the optimal reaction conditions.

The generality of the optimized protocol was then tested by the synthesis of a range of functionalized benzo[h]quinolines (Scheme 1 ). We have used pyrans functionalized with different secondary amine and aryl groups and the yield was generally not affected significantly by their nature, although the presence of a 2-thienyl group lowered the yield of product.

The structure of one of the products ( $\mathbf{3 g}$ ) was confirmed by single-crystal X-ray analysis (Figure 1 ). ${ }^{35}$ It was found that benzo[h]quinoline ring is completely planar and one unit cell contains eight molecules. It is interesting to note that the C8-C7-C4 angle is $115.29^{\circ}$ and not $120^{\circ}$, probably due to steric repulsion by the piperidine ring at $\mathrm{C} 17$. Bond angles for C12-C7-C4, C18-C17-N4 are $124.81^{\circ}$ and $120.76^{\circ}$, respectively. This repulsion also leads to a higher torsion angle of $59^{\circ}$ between the benzo[ $h$ ]quinoline ring and the $p$ fluorophenyl ring.
Table 1 Optimization of Reaction Conditions ${ }^{\mathrm{a}}$

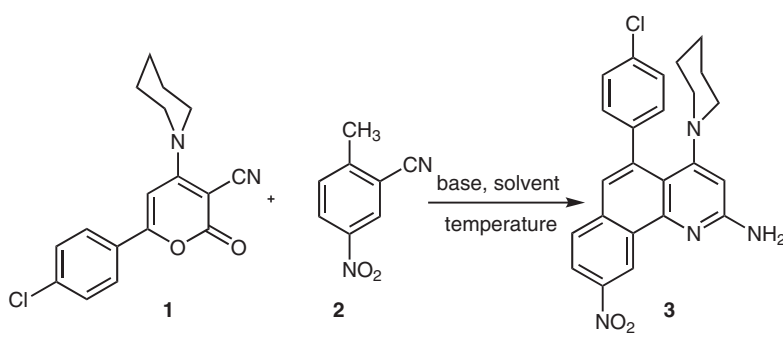

\begin{tabular}{|c|c|c|c|c|c|}
\hline Entry & Base & Solvent & $\operatorname{Temp}\left({ }^{\circ} \mathrm{C}\right)$ & $t(\mathrm{~h})$ & Yield $3(\%)^{c}$ \\
\hline 1 & $\mathrm{NEt}_{3}$ & DMSO & 100 & 12 & $-^{\mathrm{d}}$ \\
\hline 2 & $\mathrm{NEt}_{3}$ & DMF & 100 & 12 & $--^{d}$ \\
\hline 3 & $\mathrm{NaNH}_{2}$ & DMSO & r.t. ${ }^{b}$ & 12 & $--^{d}$ \\
\hline 4 & $\mathrm{NaNH}_{2}$ & DMSO & 70 & 12 & $-^{\mathrm{d}}$ \\
\hline 5 & $\mathrm{NaNH}_{2}$ & DMF & 70 & 12 & $--^{d}$ \\
\hline 6 & $\mathrm{NaNH}_{2}$ & DMF & 100 & 12 & $--^{d}$ \\
\hline 7 & $\mathrm{NaH}$ & DMF & r.t. & 12 & $-^{e}$ \\
\hline 8 & $\mathrm{NaH}$ & DMF & 100 & 12 & $-\mathrm{e}$ \\
\hline 9 & $\mathrm{NaH}$ & DMSO & 100 & 12 & $-\mathrm{e}$ \\
\hline 10 & $\mathrm{KOH}$ & DMF & r.t. & 12 & 45 \\
\hline 11 & $\mathrm{KOH}$ & DMF & 100 & 6 & 93 \\
\hline 12 & $\mathrm{KOH}$ & DMSO & 100 & 12 & 65 \\
\hline 13 & $t$-BuOK & DMF & 100 & 12 & 65 \\
\hline 14 & $\mathrm{CS}_{2} \mathrm{CO}_{3}$ & DMF & 100 & 12 & $--^{d}$ \\
\hline 15 & $\mathrm{LiOH}$ & DMF & 100 & 8 & 55 \\
\hline 16 & $\mathrm{NaOH}$ & DMF & 100 & 8 & $-\mathrm{e}$ \\
\hline 17 & $\mathrm{KOH}$ & $\mathrm{H}_{2} \mathrm{O}$ & 100 & 12 & $-d$ \\
\hline 18 & $\mathrm{KOH}$ & DMSO & 100 & 6 & 40 \\
\hline
\end{tabular}

${ }^{a}$ All the reactions were performed by stirring 6-(4-chlorophenyl)-4-(piperidin-1-yl)-2H-pyran-2-one-3-carbonitrile (1; 0.5 mmol), 2-methyl-5-nitrobenzonitrile (2; $0.6 \mathrm{mmol})$, base $(1.0 \mathrm{mmol})$ in solvent $(5.0 \mathrm{~mL})$.

${ }^{b}$ Room temperature $\left(25-30^{\circ} \mathrm{C}\right)$.

c Isolated yield reported.

${ }^{\mathrm{d}}$ Starting materials recovered.

e Complex mixture formation.

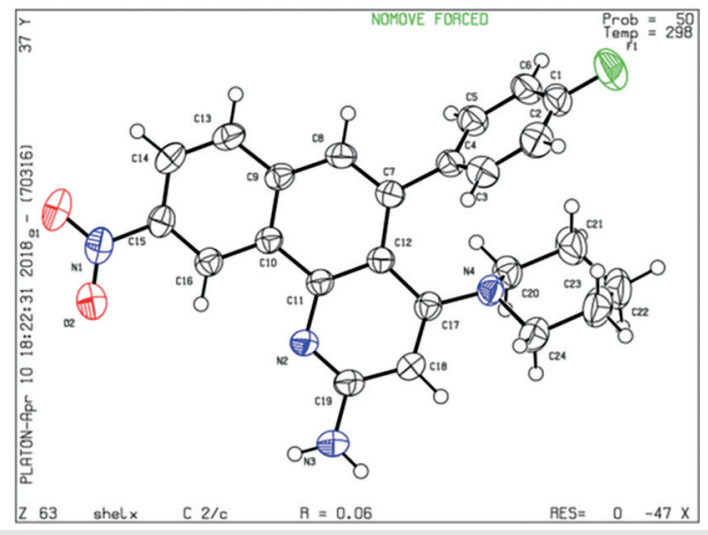

Figure 1 ORTEP diagram of $\mathbf{3 g}$ 
<smiles>[X]CCN(CC)c1cc([Al])oc(=O)c1[N+]#N</smiles><smiles>[X]CCN(CC)c1cc(N)nc2c1c(Br)cc1ccc([N+](=O)[O-])cc12</smiles><smiles>[X]CCN(CCC)c1cc(N)nc2c1c(-c1ccccc1)cc1ccc([N+](=O)[O-])cc12</smiles>

3a $\mathrm{X}=\mathrm{CH}_{2} ; 88 \%$ $3 \mathbf{b} X=0 ; 85 \%$<smiles>[X]CCN(CCC)c1cc(N)nc2c1c(-c1ccc(F)cc1)cc1ccc([N+](=O)[O-])cc12</smiles>

3g X $=\mathrm{CH}_{2} ; 81 \%$ $3 \mathrm{~h} \mathrm{X}=0 ; 80 \%$

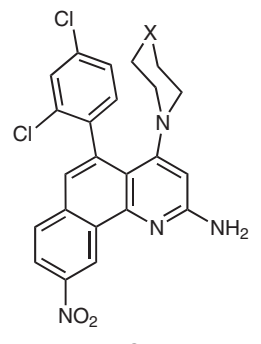

$3 \mathrm{mX}=\mathrm{CH}_{2} ; 72 \%$ 3n $\mathrm{X}=\mathrm{O} ; 74 \%$

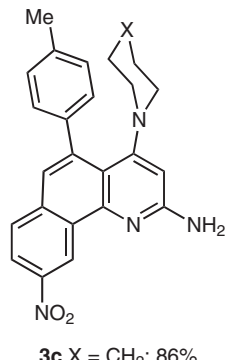

$3 c \mathrm{X}=\mathrm{CH}_{2} ; 86 \%$ $3 d X=0 ; 82 \%$

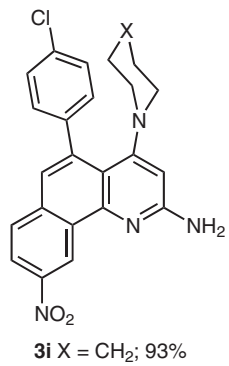
3j $\mathrm{X}=\mathrm{O} ; 91 \%$

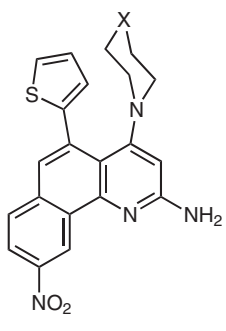

3o $\mathrm{X}=\mathrm{CH}_{2} ; 80 \%$ $3 p X=0 ; 84 \%$

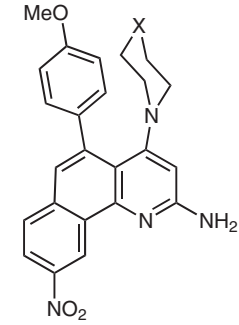

3e $\mathrm{X}=\mathrm{CH}_{2} ; 78 \%$ 3f $X=0 ; 79 \%$<smiles>[X]CCN(CC[R16])c1cc(N)nc2c1c(-c1ccc(Br)cc1)cc1ccc([N+](=O)[O-])cc12</smiles><smiles>[X]CCCN(CC[X])c1cc(N)nc2c1c(-c1ccc(OC)cc1)cc1ccc([N+](=O)[O-])cc12</smiles>

$3 q \mathrm{X}=\mathrm{N}-\mathrm{CH}_{2} \mathrm{Ph} ; 75 \%$ $3 \mathrm{r} \mathrm{X}=\mathrm{N}-\mathrm{Me} ; 72 \%$

Scheme 1 Synthesis of 2-amino-5-aryl-4-sec-amino-benzo[ $h] q u i n o-$ line-6-carbonitriles 3. Reagents and conditions: 6 -aryl-4-sec-amino- $2 \mathrm{H}$ pyran-2-one-3-carbonitriles 1 ( $0.5 \mathrm{mmol}), 2$-methyl-5-nitrobenzonitrile $2(0.6 \mathrm{mmol}), \mathrm{KOH}(1.0 \mathrm{mmol})$ in DMF $(5.0 \mathrm{~mL})$. Isolated yield reported.

To expand the scope of the reaction, 6-aryl-4-methylsulfanyl-2-oxo-2H-pyran-3-carbonitriles 4 were reacted under similar reaction conditions with $\mathbf{2}$. The usual workup and purification afforded the 6-aryl-4-(2-cyano-4-nitrobenzyl)-2-oxo-2H-pyran-3-carbonitriles 5a-d chemoselectively (Scheme 2). Interestingly, it was observed that the presence of a thiomethyl group at C-4 diverts the chemoselectivity and provides 6-aryl-4-(2-cyano-4-nitrobenzyl)-2oxo-2H-pyran-3-carbonitriles exclusively, without forma-

tion of benzo[ $h]$ quinolines. The structures of these condensation products were confirmed unambiguously by NMR and IR spectroscopy and by HRMS.
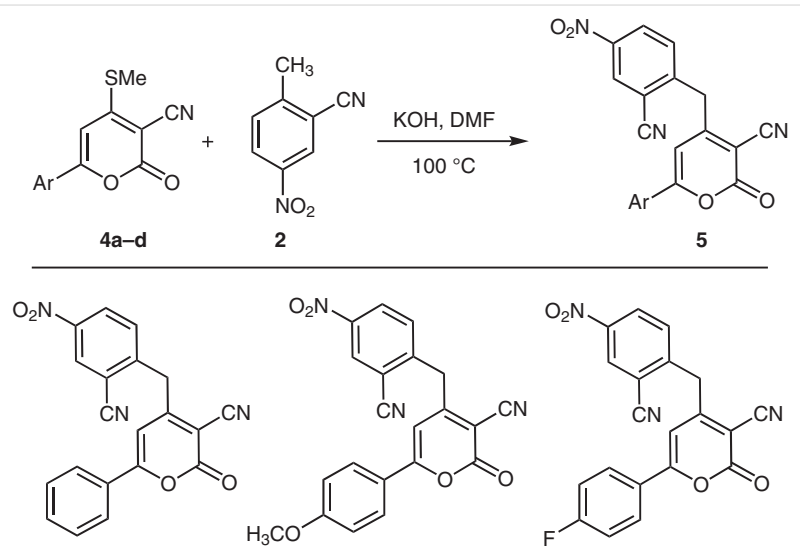

5a $71 \%$

5b $75 \%$

5c $74 \%$<smiles>N#Cc1cc([N+](=O)[O-])ccc1Cc1cc(-c2ccc(Cl)cc2)oc(=O)c1C#N</smiles>

$5 d 74 \%$

Scheme 2 Synthesis of 6-aryl-4-(2-cyano-4-nitrobenzyl)-2-oxo-2Hpyran-3-carbonitrile $\mathbf{5 a}$ - $\mathbf{d}$

Mechanistically, the reaction is probably initiated by the generation of the carbanion of 2-methyl-5-nitrobenzonitrile (Scheme 3 ), which reacts at C-4 or C-6 of the 2-pyranone, depending on the substrate selected. If 6-aryl-4-secamino-2-oxo-2H-pyran-3-carbonitriles are the substrate, the carbanion attacks at C-6 through Michael addition to afford intermediate $\mathbf{A}$. The latter intermediate can then undergo ring opening and protonation to afford two possible intermediate regioisomers, $\mathbf{B}$ and $\mathbf{D}$. If reaction proceeds via cis intermediate $\mathbf{B}$, then, in the presence of excess base, the benzylic carbanion attacks the aromatic nitrile group to afford intermediate $\mathbf{C}$. Intermediate $\mathbf{C}$ undergoes decarboxylation followed by cyclization onto the aliphatic nitrile group with tautomerization to afford the benzo[ $h]$ quinolines 3.

Alternatively, if intermediate $\mathbf{D}$ were to be formed, then benzylic carbanion generated by the excess base could cyclize by involvement of vinylic nitrile to provide intermediate $\mathbf{E}$. Subsequently, the imine group of intermediate $\mathbf{E}$ can cyclize onto the aromatic nitrile followed by decarboxylation to afford $\mathbf{F}$, which, on tautomerization, provides functionalized phenanthridines. However, the reaction provides benzo[ $h]$ quinolines chemoselectively without any trace of phenanthridine. 

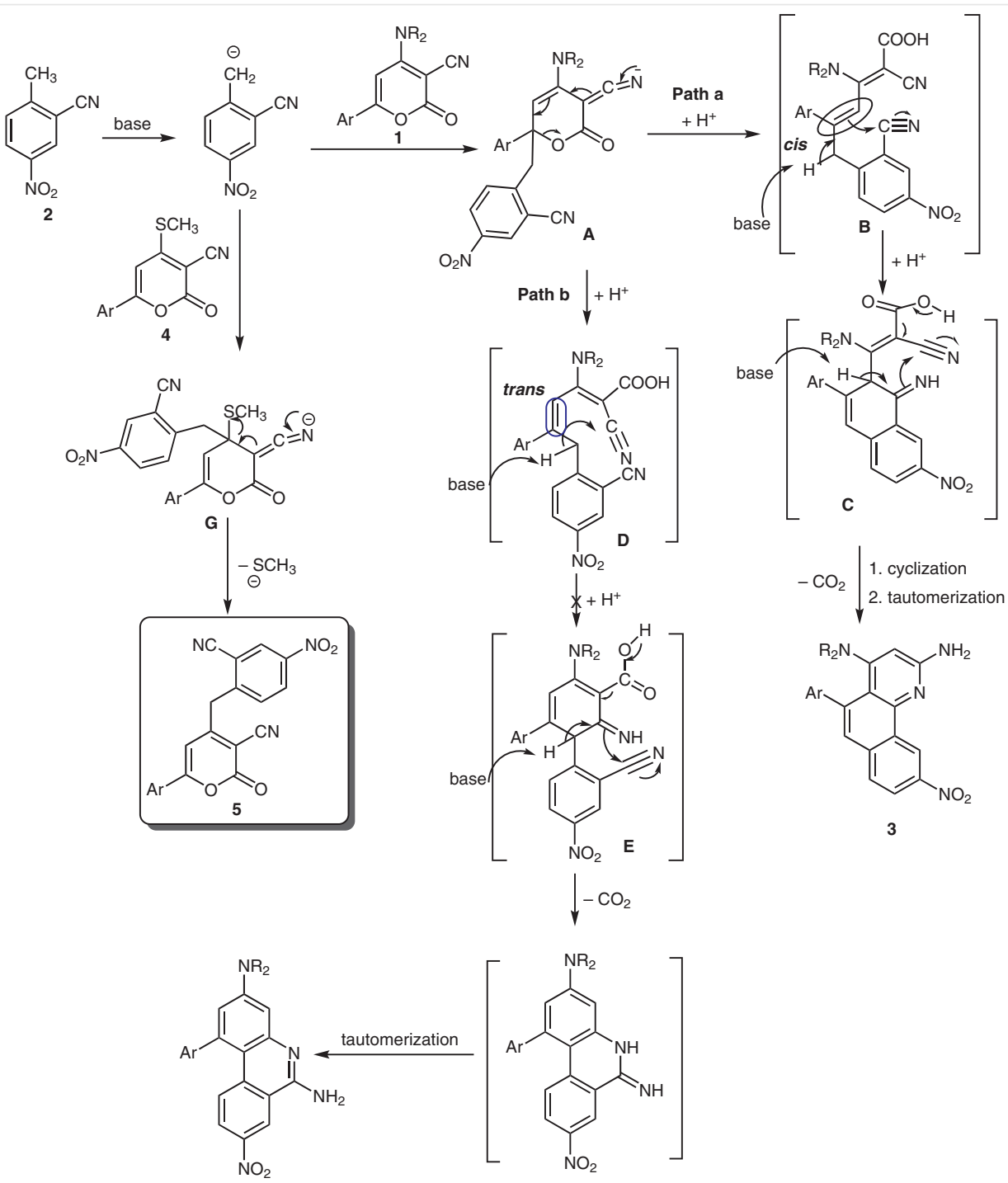

Scheme 3 Plausible mechanism for the synthesis of 2-amino-5-aryl-4- sec-amino-benzo[h]quinoline-6-carbonitriles and 6-aryl-4-(2-cyano-4-nitrobenzyl)-2-oxo-2H-pyran-3-carbonitriles

On the other hand, if 6-aryl-4-methylsulfanyl-2-oxo$2 \mathrm{H}$-pyran-3-carbonitriles are used as precursors then the carbanion from 2-methyl-5-nitrobenzonitrile attacks at C-4 of the pyran ring via Michael addition with formation of intermediate $\mathbf{G}$. Loss of the methylsulfanyl group then affords the 6-aryl-4-(2-cyano-4-nitrobenzyl)-2-oxo-2H-pyran-3carbonitriles 5 exclusively.

It is interesting to note that, if 6-aryl-4-methylsulfanyl2-oxo-2H-pyran-3-carbonitriles were used as precursors, the carbanion generated from 2-methyl-5-nitrotoluene attacks at C-4 rather than C-6, possibly due to the greater electrophilicity at C-4 caused by the vacant d-orbitals of sulfur and the better leaving group ability of the SMe group compared with the secondary amine.

To probe the mechanism, we performed the reaction of 2-oxo-4-piperidin-1-yl-6-thiophen-2-yl-2H-pyran-3-carbonitrile and 2-methyl-5-nitrotoluene using a lower concentration of base and isolated the 3-(1-amino-7-nitro-3thiophen-2-yl-naphthalen-2-yl)-3-(piperidin-1-yl)-acrylonitrile in $81 \%$ yield, which supports the intermediacy of $\mathbf{C}$ (Scheme 4). Furthermore, we used compound 7 and performed the cyclization under optimized reaction conditions and isolated the benzo[ $h]$ quinoline. 
<smiles>N#Cc1c(N2CCCCC2)cc(-c2cccs2)oc1=O</smiles><smiles>Cc1ccc([N+](=O)[O-])cc1C#N</smiles><smiles>CCCN(CCC)c1cc(N)nc2c1c(-c1cccs1)cc1ccc([N+](=O)[O-])cc12</smiles>

Scheme 4 Synthesis of 3-(1-amino-7-nitro-3-(thiophene-2-yl)naphthalene-2-yl)-3-(piperidin-1-yl)acrylonitrile (7) and benzo[h]quinoline (3o)

It is interesting to note that reaction of 2-pyranone with 2-cyanomethylbenzonitrile ${ }^{30}$ requires 35-50 hours for completion of the reaction, whereas the use of 2-methyl-5nitrobenzonitrile as a carbanion source provides the ben$z o[h]$ quinolines in 4-6 hours. We propose that the presence of a nitrile group reduces the rate of reaction, probably by stabilizing the carbanion formed and slowing the cyclization step due to steric hindrance.

In summary, we have developed a simple and efficient approach for the chemoselective synthesis of 5-aryl-4-secamino-benzo[h]quinoline-6-carbonitriles 3 and 6-aryl-4(2-cyano-4-nitrobenzyl)-2-oxo-2H-pyran-3-carbonitriles 5 by reaction of 2-methyl-5-nitrobenzonitrile with 6-aryl-4sec-amino-2H-pyran-2-one-3-carbonitriles 1 and 6-aryl-4methylsulfanyl-2H-pyran-2-one-3-carbonitriles 4, respectively, under basic conditions. We also investigated mechanistic aspects of the reaction and found that the reaction can be stopped at the functionalized naphthalene stage. We confirmed the structure of compound $\mathbf{3 g}$ by single-crystal $\mathrm{X}$-ray analysis and found that the aryl group present at position 5 is distorted from the usual $\mathrm{sp}^{2}$ angle of $120^{\circ}$.

Commercially available reagents were used directly. NMR spectra were recorded at $400 \mathrm{MHz}$ for ${ }^{1} \mathrm{H}$ and $100 \mathrm{MHz}$ for ${ }^{13} \mathrm{C}$ and chemical shifts $(\delta)$ are given as parts per million. Spectra were referenced to the residual ${ }^{1} \mathrm{H}$ signal of $\mathrm{CDCl}_{3}$ at $\delta=7.24 \mathrm{ppm},{ }^{13} \mathrm{C}$ of $\mathrm{CDCl}_{3}$ at $\delta=$ $77.00 \mathrm{ppm}$, the residual ${ }^{1} \mathrm{H}$ of DMSO- $d_{6}$ at $\delta=2.49 \mathrm{ppm}$ and the ${ }^{13} \mathrm{C}$ of of DMSO- $d_{6}$ at $\delta=39.50 \mathrm{ppm}$ as the internal standards. Splitting patterns in ${ }^{1} \mathrm{H}$ NMR data are described as s, singlet; d, doublet; dd, double doublet; $t$, triplet; bs, broad singlet; $m$, multiplet and coupling constants given in hertz (Hz). HRMS was recorded using an Agilent LCMS with Quadropole time of flight using the ESI mode of ionization. All the required starting materials were synthesized by following reported procedures. ${ }^{33 \mathrm{a}-\mathrm{c}}$
Synthesis of Benzo[ $h]$ quinolines (3a-p); General Procedure

A mixture of 6-aryl-2-oxo-4-sec-amino-2H-pyran-3-carbonitrile $\mathbf{1}$ (0.5 mmol), 2-methyl-5-nitrobenzonitrile $2(0.6 \mathrm{mmol})$ and $\mathrm{KOH}(1.0$ $\mathrm{mmol})$ in DMF $\left(5.0 \mathrm{~mL}\right.$ ) was stirred at $100-110^{\circ} \mathrm{C}$ for $6 \mathrm{~h}$. After completion of reaction, the reaction mixture was poured onto crushed ice and neutralized with $10 \% \mathrm{HCl}$ to $\mathrm{pH} \sim 6-7$. The solid product was filtered, washed with cold water, dried and purified by silica gel column chromatography, eluting with hexane-EtOAc (70:30).

Synthesis of 6-Aryl-4-(2-cyano-4-nitrobenzyl)-2-oxo-2H-pyran-3carbonitriles (5a-d); General Procedure

A mixture of 6-aryl-4-(methylthio)-2-oxo-2H-pyran-3-carbonitrile 4 (0.5 mmol), 2-methyl-5-nitrobenzonitrile $2(0.6 \mathrm{mmol})$ and $\mathrm{KOH}(1.0$ $\mathrm{mmol})$ in DMF $(5.0 \mathrm{~mL})$ was stirred at $100-110^{\circ} \mathrm{C}$ for $1 \mathrm{~h}$. When the reaction was complete, the mixture was poured onto crushed ice and neutralized with $10 \% \mathrm{HCl}$ to $\mathrm{pH} \sim 6-7$. The solid product was filtered, washed with water, dried, and purified by silica gel column chromatography, eluting with hexane-EtOAc (60:40).

3-(1-Amino-7-nitro-3-(thiophen-2-yl)naphthalen-2-yl)-3-(piperidin-1-yl)acrylonitrile (7)

A mixture of 2-oxo-4-(piperidin-1-yl)-6-(thiophen-2-yl)-2H-pyran3-carbonitrile $10(0.5 \mathrm{mmol}), 2-2-$ methyl-5-nitrobenzonitrile 1 $(0.6 \mathrm{mmol})$, and $\mathrm{KOH}(0.6 \mathrm{mmol})$ in $\operatorname{DMF}(5.0 \mathrm{~mL})$ was stirred at $100-$ $110{ }^{\circ} \mathrm{C}$ for $2-3 \mathrm{~h}$. After completion of reaction, the reaction mixture was poured onto crushed ice followed by neutralization with $10 \% \mathrm{HCl}$ to $\mathrm{pH} \sim 6-7$. The crude material was filtered, washed with water, dried, and purified by silica gel column chromatography eluting with hexane-EtOAc (70:30).

\section{9-Nitro-5-phenyl-4-(piperidin-1-yl)benzo[h]quinolin-2-amine}

(3a)

Yield: 88\% (175 mg); red solid; mp 190-192 ${ }^{\circ} \mathrm{C}$.

IR (KBr): 3356, $1334 \mathrm{~cm}^{-1}$.

$\left.{ }^{1} \mathrm{H} \mathrm{NMR} \mathrm{(400} \mathrm{MHz,} \mathrm{CDCl}_{3}\right): \delta=0.75-0.85(\mathrm{~m}, 2 \mathrm{H}), 0.92-1.00(\mathrm{~m}, 2 \mathrm{H})$, 1.21-1.26 (m, $2 \mathrm{H}), 2.33-2.39\left(\mathrm{~m}, 2 \mathrm{H}, \mathrm{CH}_{2}-\mathrm{N}-\mathrm{CH}_{2}\right), 2.97$ (d, $J=12 \mathrm{~Hz}$, $\left.2 \mathrm{H}, \mathrm{CH}_{2}-\mathrm{N}-\mathrm{CH}_{2}\right), 5.22\left(\mathrm{~s}, 2 \mathrm{H},-\mathrm{NH}_{2}\right), 6.30(\mathrm{~s}, 1 \mathrm{H}, \mathrm{ArH}), 7.25-7.32(\mathrm{~m}$, $3 \mathrm{H}, \operatorname{ArH}), 7.36(\mathrm{~s}, 1 \mathrm{H}, \operatorname{ArH}), 7.41(\mathrm{~d}, J=8 \mathrm{~Hz}, 2 \mathrm{H}, \operatorname{ArH}), 7.76(\mathrm{~d}, J=$ $8 \mathrm{~Hz}, 1 \mathrm{H}, \mathrm{ArH}), 8.24$ (dd, J = $8 \mathrm{~Hz}, 1 \mathrm{H}, \mathrm{ArH}), 9.96$ (s, $1 \mathrm{H}, \mathrm{ArH})$.

${ }^{13} \mathrm{C}$ NMR $\left(100 \mathrm{MHz}, \mathrm{CDCl}_{3}\right): \delta=23.5,24.3,52.5,98.5,113.1,121.2$, $122.0,124.5,127.0,127.2,128.3,129.1,129.4,136.3,141.5,141.6$, $145.2,148.5,157.7,160.2$.

HRMS (ESI): $m / z[M H]^{+}$calcd. for $\mathrm{C}_{24} \mathrm{H}_{23} \mathrm{~N}_{4} \mathrm{O}_{2}$ : 399.1816; found: 399.1819 .

4-Morpholino-9-nitro-5-phenylbenzo[ $h]$ quinolin-2-amine (3b) Yield: $85 \%(170 \mathrm{mg})$; red solid; $\mathrm{mp}>250^{\circ} \mathrm{C}$.

IR (KBr): 3449, $1334 \mathrm{~cm}^{-1}$.

${ }^{1} \mathrm{H} \mathrm{NMR}\left(400 \mathrm{MHz}, \mathrm{CDCl}_{3}\right): \delta=2.52(\mathrm{t}, J=20 \mathrm{~Hz}, 2 \mathrm{H}), 2.66(\mathrm{t}, J=20 \mathrm{~Hz}$, $2 \mathrm{H}), 2.92(\mathrm{~d}, J=12 \mathrm{~Hz}, 2 \mathrm{H}), 3.49(\mathrm{~d}, J=12 \mathrm{~Hz}, 2 \mathrm{H}), 4.91\left(\mathrm{~s}, 2 \mathrm{H},-\mathrm{NH}_{2}\right)$, 6.34 (s, $1 \mathrm{H}, \mathrm{ArH}), 7.40-7.42(\mathrm{~m}, 2 \mathrm{H}, \mathrm{ArH}), 7.45$ (s, $1 \mathrm{H}, \mathrm{ArH}), 7.48-$ $7.50(\mathrm{~m}, 3 \mathrm{H}, \mathrm{ArH}), 7.86(\mathrm{~d}, J=8 \mathrm{~Hz}, 1 \mathrm{H}, \mathrm{ArH}), 8.34(\mathrm{dd}, J=12 \mathrm{~Hz}, 1 \mathrm{H}$, ArH), 10.0-10.0 (m, $1 \mathrm{H}, \mathrm{ArH})$.

${ }^{13} \mathrm{C}$ NMR $\left(100 \mathrm{MHz}\right.$, DMSO- $\left.d_{6}\right): \delta=51.1,64.6,99.2,111.4,120.8$, 121.0, 123.3, 127.0, 127.1, 128.6, 129.1, 129.3, 136.0, 141.0, 141.7, 144.4, 148.8, 157.8, 159.3.

HRMS (ESI): $m / z[M H]^{+}$calcd. for $\mathrm{C}_{23} \mathrm{H}_{21} \mathrm{~N}_{4} \mathrm{O}_{3}$ : 401.1608; found: 401.1609. 


\section{9-Nitro-4-(piperidin-1-yl)-5-(p-tolyl)benzo[ $h]$ quinolin-2-amine} (3c) Yield: 86\% (180 mg); red solid; $\mathrm{mp} 150-152^{\circ} \mathrm{C}$.

IR (KBr): 3480, $1337 \mathrm{~cm}^{-1}$.

${ }^{1} \mathrm{H}$ NMR (400 MHz, $\mathrm{CDCl}_{3}$ ): $\delta=1.18-1.26(\mathrm{~m}, 3 \mathrm{H}), 1.39-1.40(\mathrm{~m}, 3 \mathrm{H})$, $2.33\left(\mathrm{~s}, 3 \mathrm{H},-\mathrm{CH}_{3}\right), 2.78-2.90\left(\mathrm{~m}, 3 \mathrm{H}, \mathrm{CH}_{2}-\mathrm{N}_{-} \mathrm{CH}_{2}\right), 3.01-3.09(\mathrm{M}, 1 \mathrm{H}$, $\left.\mathrm{CH}_{2}-\mathrm{N}-\mathrm{CH}_{2}\right), 4.84\left(\mathrm{~s}, 2 \mathrm{H},-\mathrm{NH}_{2}\right), 7.13(\mathrm{~d}, J=8 \mathrm{~Hz}, 2 \mathrm{H}, \mathrm{ArH}), 7.19(\mathrm{~s}$, $1 \mathrm{H}, \mathrm{ArH}), 7.23$ (s, $1 \mathrm{H}, \mathrm{ArH}), 7.29(\mathrm{~d}, J=8 \mathrm{~Hz}, 2 \mathrm{H}, \mathrm{ArH}), 7.8(\mathrm{~d}, J=8 \mathrm{~Hz}$, $1 \mathrm{H}, \mathrm{ArH}), 8.16(\mathrm{~d}, J=8 \mathrm{~Hz}, 1 \mathrm{H}, \mathrm{ArH}), 8.81(\mathrm{~s}, 1 \mathrm{H}, \mathrm{ArH})$.

${ }^{13} \mathrm{C}$ NMR $\left(100 \mathrm{MHz}, \mathrm{CDCl}_{3}\right): \delta=21.2,23.6,24.3,52.5,98.3,113.2$, 121.2, 122.0, 124.2, 127.8, 128.2, 129.2, 129.3, 136.4, 136.8, 138.8, 141.6, 145.1, 148.7, 157.7, 160.1 .

HRMS (ESI): $m / z[M H]^{+}$calculated for $\mathrm{C}_{25} \mathrm{H}_{25} \mathrm{~N}_{4} \mathrm{O}_{2}$ : 418.1972; found: 418.1973.

\section{4-Morpholino-9-nitro-5-( $p$-tolyl)benzo[ $h$ ]quinolin-2-amine (3d) Yield: $82 \%$ (170 mg); red solid; mp 240-242 ${ }^{\circ} \mathrm{C}$. \\ IR (KBr): $3354,1332 \mathrm{~cm}^{-1}$. \\ ${ }^{1} \mathrm{H} \mathrm{NMR}\left(400 \mathrm{MHz}, \mathrm{CDCl}_{3}\right): \delta=2.44\left(\mathrm{~s}, 3 \mathrm{H},-\mathrm{CH}_{3}\right), 2.54-2.62(\mathrm{~m}, 2 \mathrm{H})$, 2.65-2.69 (m, $2 \mathrm{H}), 2.93$ (d, $\left.J=8 \mathrm{~Hz}, 2 \mathrm{H}, \mathrm{CH}_{2}-\mathrm{N}-\mathrm{CH}_{2}\right), 3.5(\mathrm{~d}, J=8 \mathrm{~Hz}$, $\left.2 \mathrm{H}, \mathrm{CH}_{2}-\mathrm{N}-\mathrm{CH}_{2}\right), 4.88\left(\mathrm{~s}, 2 \mathrm{H},-\mathrm{NH}_{2}\right), 6.32(\mathrm{~s}, 1 \mathrm{H}, \mathrm{ArH}), 7.22(\mathrm{~d}, J=$ $8 \mathrm{~Hz}, 2 \mathrm{H}, \mathrm{ArH}$ ), 7.37 (d, J = 4 Hz, $2 \mathrm{H}, \mathrm{ArH}), 7.43$ (s, $1 \mathrm{H}, \mathrm{ArH}), 7.84$ (d, $J=8 \mathrm{~Hz}, 1 \mathrm{H}, \mathrm{ArH}), 8.33(\mathrm{dd}, J=8 \mathrm{~Hz}, 1 \mathrm{H}, \mathrm{ArH}), 10.01-10.02(\mathrm{~m}, 1 \mathrm{H}$, $\mathrm{ArH})$.}

${ }^{13} \mathrm{C}$ NMR $\left(100 \mathrm{MHz}, \mathrm{CDCl}_{3}\right): \delta=21.7,52.1,70.0,114.6,116.0,121.0$, 122.5, 125.2, 127.0, 127.1, 127.4, 127.5, 128.4, 129.7, 130.1, 130.2, $130.8,130.8,133.1,156.4$.

HRMS (ESI): $m / z$ [MH] ${ }^{+}$calcd. for $\mathrm{C}_{24} \mathrm{H}_{23} \mathrm{~N}_{4} \mathrm{O}_{3}$ : 415.1765; found: 415.1762 .

\section{5-(4-Methoxyphenyl)-9-nitro-4-(piperidin-1-yl)benzo[h]quino-} lin-2-amine (3e)

Yield: $78 \%$ (167 mg); red solid; $\mathrm{mp} 245-257^{\circ} \mathrm{C}$.

IR (KBr): $3401,1333 \mathrm{~cm}^{-1}$.

${ }^{1} \mathrm{H} \mathrm{NMR}\left(400 \mathrm{MHz}, \mathrm{CDCl}_{3}\right): \delta=0.81-0.86(\mathrm{~m}, 2 \mathrm{H}), 1.20-1.31(\mathrm{~m}, 4 \mathrm{H})$, 2.38-2.43 (m, $\left.2 \mathrm{H}, \mathrm{CH}_{2}-\mathrm{N}-\mathrm{CH}_{2}\right), 3.01-3.04\left(\mathrm{~m}, 2 \mathrm{H}, \mathrm{CH}_{2}-\mathrm{N}-\mathrm{CH}_{2}\right), 3.85$ (s, $3 \mathrm{H},-\mathrm{OMe}), 5.27\left(\mathrm{~s}, 2 \mathrm{H},-\mathrm{NH}_{2}\right), 6.31(\mathrm{~s}, 1 \mathrm{H}, \mathrm{ArH}), 6.9(\mathrm{~d}, J=8 \mathrm{~Hz}$, $2 \mathrm{H}, \mathrm{ArH}), 7.36(\mathrm{~d}, J=8 \mathrm{~Hz}, 2 \mathrm{H}, \operatorname{ArH}), 7.39(\mathrm{~s}, 1 \mathrm{H}, \operatorname{ArH}), 7.77(\mathrm{~d}, J=$ $8 \mathrm{~Hz}, 1 \mathrm{H}, \mathrm{ArH}), 8.26$ (dd, $J=8 \mathrm{~Hz}, 1 \mathrm{H}, \mathrm{ArH}), 9.94-9.95$ (m, $1 \mathrm{H}, \mathrm{ArH}$ ).

${ }^{13} \mathrm{C}$ NMR $\left(100 \mathrm{MHz}, \mathrm{CDCl}_{3}\right): \delta=24.4,29.6,52.4,55.4,98.1,112.6$, 113.0, 121.2, 122.0, 124.1, 128.1, 128.3, 130.4, 130.5, 134.2, 136.4, 141.1, 145.0, 157.6, 159.0, 160.1 .

HRMS (ESI): $m / z[M H]^{+}$calcd. for $\mathrm{C}_{25} \mathrm{H}_{25} \mathrm{~N}_{4} \mathrm{O}_{3}$; 429.1921; found: 429.1929 .

\section{5-(4-Methoxyphenyl)-4-morpholino-9-nitrobenzo[h]quinolin-2- amine (3f)}

Yield: $79 \%$ (170 mg); red solid; $\mathrm{mp}>250{ }^{\circ} \mathrm{C}$.

IR (KBr): 3383, $1332 \mathrm{~cm}^{-1}$.

${ }^{1} \mathrm{H} \mathrm{NMR}\left(400 \mathrm{MHz}, \mathrm{CDCl}_{3}\right): \delta=2.59-2.69(\mathrm{~m}, 2 \mathrm{H}), 2.91-2.93(\mathrm{~m}, 2 \mathrm{H})$, 3.49-3.51 (m, $2 \mathrm{H}$ ), 3.86 (s, $3 \mathrm{H},-\mathrm{OMe}), 4.90$ (s, $2 \mathrm{H},-\mathrm{NH}_{2}$ ), 6.30 (s, $1 \mathrm{H}$, ArH), 6.93 (d, $J=8 \mathrm{~Hz}, 2 \mathrm{H}, \mathrm{ArH}), 7.37-7.40(\mathrm{~m}, 3 \mathrm{H}, \mathrm{ArH}), 7.81(\mathrm{~d}, J=$ $8 \mathrm{~Hz}, 1 \mathrm{H}, \mathrm{ArH}), 8.3$ (dd, J = $8 \mathrm{~Hz}, 1 \mathrm{H}, \mathrm{ArH}), 9.97-9.98(\mathrm{~m}, 1 \mathrm{H}, \mathrm{ArH})$.

${ }^{13} \mathrm{C}$ NMR $\left(100 \mathrm{MHz}\right.$, DMSO- $\left.d_{6}\right): \delta=51.3,55.7,65.2,99.4,112.0,113.0$, $121.1,121.5,123.1,128.1,129.0,129.1,129.7,131.0,134.4,139.2$, $141.1,149.4,159.2,159.8$.
HRMS (ESI): $m / z$ [MH] $]^{+}$calcd. for $\mathrm{C}_{24} \mathrm{H}_{23} \mathrm{~N}_{4} \mathrm{O}_{4}, 431.1714$; found: 431.1715.

\section{5-(4-Fluorophenyl)-9-nitro-4-(piperidin-1-yl)benzo[h]quinolin- 2-amine (3g)}

Yield: 81\% (168 mg); red solid; mp 200-202 ${ }^{\circ} \mathrm{C}$.

IR (KBr): $3358,1334 \mathrm{~cm}^{-1}$.

${ }^{1} \mathrm{H} \mathrm{NMR}\left(400 \mathrm{MHz}, \mathrm{CDCl}_{3}\right): \delta=1.19-1.23(\mathrm{~m}, 3 \mathrm{H}), 1.30-1.34(\mathrm{~m}, 3 \mathrm{H})$, 2.37-2.43 (m, $2 \mathrm{H}, \mathrm{CH}_{2}-\mathrm{N}-\mathrm{CH}_{2}$ ), 3.01-3.04 (m, $\left.2 \mathrm{H}, \mathrm{CH}_{2}-\mathrm{N}-\mathrm{CH}_{2}\right), 4.83$ (s, $\left.2 \mathrm{H},-\mathrm{NH}_{2}\right), 6.33(\mathrm{~s}, 1 \mathrm{H}, \mathrm{ArH}), 7.04-7.09(\mathrm{~m}, 2 \mathrm{H}, \mathrm{ArH}), 7.37(\mathrm{~s}, 1 \mathrm{H}$, ArH), 7.43-7.46 (m, $2 \mathrm{H}, \mathrm{ArH}), 7.82(\mathrm{~d}, J=12 \mathrm{~Hz}, 1 \mathrm{H}), 8.31$ (dd, $J=$ $8 \mathrm{~Hz}, 1 \mathrm{H}), 7.98(\mathrm{~d}, J=4 \mathrm{~Hz}, 1 \mathrm{H})$.

${ }^{13} \mathrm{C}$ NMR $\left(100 \mathrm{MHz}, \mathrm{CDCl}_{3}\right): \delta=24.6,29.7,52.7,98.8,114.1\left(\mathrm{~d}, J_{\mathrm{C}-\mathrm{F}}=\right.$ $20 \mathrm{~Hz}), 121.7\left(\mathrm{~d}, J_{\mathrm{C}-\mathrm{F}}=80 \mathrm{~Hz}\right), 124.5,128.3,129.8,131.0\left(\mathrm{~d}, J_{\mathrm{C}-\mathrm{F}}=\right.$ $10 \mathrm{~Hz}), 136.3,138.0,140.4,145.3,149.4,158.0,160.1$.

HRMS (ESI): $m / z$ [MH] $]^{+}$calcd. for $\mathrm{C}_{24} \mathrm{H}_{22} \mathrm{FN}_{4} \mathrm{O}_{2}$ : 417.1721; found: 417.1724.

\section{5-(4-Fluorophenyl)-4-morpholino-9-nitrobenzo[h]quinolin-2-} amine (3h)

Yield: $80 \%$ ( $167 \mathrm{mg}$ ); red solid; $\mathrm{mp}>250{ }^{\circ} \mathrm{C}$.

IR (KBr): $3484,1332 \mathrm{~cm}^{-1}$.

${ }^{1} \mathrm{H}$ NMR (400 MHz, $\mathrm{CDCl}_{3}$ ): $\delta=2.53-2.69(\mathrm{~m}, 4 \mathrm{H}), 2.89(\mathrm{~d}, J=8 \mathrm{~Hz}$, $\left.2 \mathrm{H}, \mathrm{CH}_{2}-\mathrm{N}-\mathrm{CH}_{2}\right), 3.52$ (d, $\left.\mathrm{J}=12 \mathrm{~Hz}, 2 \mathrm{H}, \mathrm{CH}_{2}-\mathrm{N}-\mathrm{CH}_{2}\right), 4.90(\mathrm{~s}, 2 \mathrm{H}$, $\left.\mathrm{NH}_{2}\right), 6.32(\mathrm{~s}, 1 \mathrm{H}, \mathrm{ArH}), 7.1(\mathrm{t}, J=12 \mathrm{~Hz}, 2 \mathrm{H}, \mathrm{ArH}), 7.38(\mathrm{~s}, 1 \mathrm{H}, \mathrm{ArH})$, 7.42-7.46 (m, $2 \mathrm{H}, \mathrm{ArH}), 7.84(\mathrm{~d}, J=8 \mathrm{~Hz}, 1 \mathrm{H}, \mathrm{ArH}), 8.31(\mathrm{dd}, J=4 \mathrm{~Hz}$, $1 \mathrm{H}), 9.98$ (d, $J=4 \mathrm{~Hz}, 1 \mathrm{H})$.

${ }^{13} \mathrm{C}$ NMR $\left(100 \mathrm{MHz}, \mathrm{CDCl}_{3}\right): \delta=51.3,65.4,98.5,112.5,114.2\left(\mathrm{~d}, J_{\mathrm{C}-\mathrm{F}}=\right.$ $30 \mathrm{~Hz}), 121.6\left(\mathrm{~d}, J_{\mathrm{C}-\mathrm{F}}=20 \mathrm{~Hz}\right), 125.0,128.5,130.8,130.8,136.2,139.6$, 145.4, 157.7, 159.0, 161.1, 163.5 .

HRMS (ESI): $m / z[\mathrm{MH}]^{+}$calcd. for $\mathrm{C}_{23} \mathrm{H}_{20} \mathrm{FN}_{4} \mathrm{O}_{3}: 419.1514$; found: 419.1513 .

\section{5-(4-Chlorophenyl)-9-nitro-4-(piperidin-1-yl)benzo[ $h]$ quinolin- 2-amine (3i)}

Yield: 93\% (201 mg); red solid; $\mathrm{mp} 215-217^{\circ} \mathrm{C}$.

IR (KBr): 3489, $1328 \mathrm{~cm}^{-1}$.

${ }^{1} \mathrm{H}$ NMR $\left(400 \mathrm{MHz}, \mathrm{CDCl}_{3}\right): \delta=0.94-1.02(\mathrm{~m}, 1 \mathrm{H}), 1.17-1.20(\mathrm{~m}, 1 \mathrm{H})$, $1.26-1.29(\mathrm{~m}, 3 \mathrm{H}), 1.38-1.41(\mathrm{~m}, 1 \mathrm{H}), 2.35\left(\mathrm{t}, J=24 \mathrm{~Hz}, 2 \mathrm{H}, \mathrm{CH}_{2}-\mathrm{N}-\right.$ $\left.\mathrm{CH}_{2}\right), 2.95\left(\mathrm{~d}, \mathrm{~J}=12 \mathrm{~Hz}, 2 \mathrm{H}, \mathrm{CH}_{2}-\mathrm{N}-\mathrm{CH}_{2}\right), 4.81\left(\mathrm{~s}, 2 \mathrm{H},-\mathrm{NH}_{2}\right), 6.28(\mathrm{~s}$, $1 \mathrm{H}, \mathrm{ArH}), 7.28-7.30(\mathrm{~m}, 3 \mathrm{H}, \mathrm{ArH}), 7.36(\mathrm{~d}, J=8 \mathrm{~Hz}, 2 \mathrm{H}, \mathrm{ArH}), 7.76$ (d, $J=8 \mathrm{~Hz}, 1 \mathrm{H}, \mathrm{ArH}), 8.25$ (dd, $J=8 \mathrm{~Hz}, 1 \mathrm{H}, \mathrm{ArH}), 9.92-9.93(\mathrm{~m}, 1 \mathrm{H}$, ArH).

${ }^{13} \mathrm{C}$ NMR $\left(100 \mathrm{MHz}, \mathrm{CDCl}_{3}\right): \delta=24.1,26.2,53.7,104.5,115.6,115.8$, $116.1,121.2,128.6,129.2,130.7,130.8,134.8,135.2,138.3,145.0$, 147.0, 158.8, 162.0, 164.3.

HRMS (ESI): $m / z$ [MH] ${ }^{+}$calcd. for $\mathrm{C}_{24} \mathrm{H}_{22} \mathrm{ClN}_{4} \mathrm{O}_{2}$ : 433.1426; found: 433.1435

\section{5-(4-Chlorophenyl)-4-morpholino-9-nitrobenzo[h]quinolin-2- amine (3j)}

Yield: 91\% (197 mg); red solid; $\mathrm{mp} 150-152{ }^{\circ} \mathrm{C}$.

IR (KBr): 3353, $1329 \mathrm{~cm}^{-1}$.

${ }^{1} \mathrm{H}$ NMR $\left(400 \mathrm{MHz}, \mathrm{CDCl}_{3}\right): \delta=2.60-2.71(\mathrm{~m}, 4 \mathrm{H}), 2.90(\mathrm{~d}, J=12 \mathrm{~Hz}$, $\left.2 \mathrm{H}, \mathrm{CH}_{2}-\mathrm{N}-\mathrm{CH}_{2}\right), 3.55\left(\mathrm{~d}, \mathrm{~J}=12 \mathrm{~Hz}, 2 \mathrm{H}, \mathrm{CH}_{2}-\mathrm{N}-\mathrm{CH}_{2}\right), 4.97(\mathrm{~s}, 2 \mathrm{H}$, $\left.-\mathrm{NH}_{2}\right), 6.35(\mathrm{~s}, 1 \mathrm{H}, \mathrm{ArH}), 7.38-7.44(\mathrm{~m}, 5 \mathrm{H}, \mathrm{ArH}), 7.85(\mathrm{~d}, J=8 \mathrm{~Hz}, 1 \mathrm{H}$, $\operatorname{ArH}), 8.34(\mathrm{dd}, J=8 \mathrm{~Hz}, 1 \mathrm{H}), 9.99(\mathrm{~d}, J=4 \mathrm{~Hz}, 1 \mathrm{H})$. 
${ }^{13} \mathrm{C}$ NMR $\left(100 \mathrm{MHz}\right.$, DMSO- $\left.d_{6}\right): \delta=55.4,67.6,112.6,116.4,120.4$, 120.7, 121.3, 121.3, 128.3, 130.4, 130.6, 133.0, 137.3, 139.5, 139.6, $142.7,144.4,145.5,160.6$.

HRMS (ESI): $m / z[M H]^{+}$calcd. for $\mathrm{C}_{23} \mathrm{H}_{20} \mathrm{ClN}_{4} \mathrm{O}_{3}$ : 435.1218; found: 435.1219

\section{5-(4-Bromophenyl)-9-nitro-4-(piperidin-1-yl)benzo[h]quinolin- 2-amine (3k)}

Yield: $82 \%$ (195 mg); red solid; $\mathrm{mp} 150-152{ }^{\circ} \mathrm{C}$.

IR (KBr): 3366, $1333 \mathrm{~cm}^{-1}$.

${ }^{1} \mathrm{H}$ NMR $\left(400 \mathrm{MHz}, \mathrm{CDCl}_{3}\right): \delta=1.15-1.27(\mathrm{~m}, 4 \mathrm{H}), 1.41-1.47(\mathrm{~m}, 2 \mathrm{H})$, 2.85-2.90 (m, $\left.2 \mathrm{H}, \mathrm{CH}_{2}-\mathrm{N}-\mathrm{CH}_{2}\right), 3.01-3.08\left(\mathrm{~m}, 2 \mathrm{H}, \mathrm{CH}_{2}-\mathrm{N}-\mathrm{CH}_{2}\right), 4.87$ $\left(\mathrm{s}, 2 \mathrm{H},-\mathrm{NH}_{2}\right), 7.19-7.20(\mathrm{~m}, 3 \mathrm{H}, \mathrm{ArH}), 7.27(\mathrm{~d}, J=12 \mathrm{~Hz}, 1 \mathrm{H}, \mathrm{ArH})$, $7.46(\mathrm{~d}, J=8 \mathrm{~Hz}, 2 \mathrm{H}), 7.80-7.81(\mathrm{~m}, 1 \mathrm{H}, \mathrm{ArH}), 8.18(\mathrm{dd}, J=8 \mathrm{~Hz}, 1 \mathrm{H})$, $8.80(\mathrm{~s}, 1 \mathrm{H}, \mathrm{ArH})$.

${ }^{13} \mathrm{C}$ NMR $\left(100 \mathrm{MHz}, \mathrm{CDCl}_{3}\right): \delta=23.5,26.0,66.3,109.0,118.8,119.2$, 120.6, 120.7, 121.6, 121.1, 130.0, 130.1, 131.0, 131.1, 136.7, 139.0, $142.0,143.0,144.6,160.0$.

HRMS (ESI): $m / z$ [MH] $]^{+}$calcd. for $\mathrm{C}_{24} \mathrm{H}_{22} \mathrm{BrN}_{4} \mathrm{O}_{2}$ : 477.0921; found: 479.0901 .

\section{5-(4-Bromophenyl)-4-morpholino-9-nitrobenzo[h]quinolin-2- amine (31)}

Yield: 83\% (199 mg); red solid; mp $210-212{ }^{\circ} \mathrm{C}$.

IR (KBr): 3351, $1338 \mathrm{~cm}^{-1}$.

${ }^{1} \mathrm{H}$ NMR (400 MHz, $\left.\mathrm{CDCl}_{3}\right): \delta=2.58-2.70(\mathrm{~m}, 4 \mathrm{H}), 2.85(\mathrm{~d}, J=12 \mathrm{~Hz}$, $\left.2 \mathrm{H}, \mathrm{CH}_{2}-\mathrm{N}-\mathrm{CH}_{2}\right), 3.54\left(\mathrm{~d}, \mathrm{~J}=8 \mathrm{~Hz}, 2 \mathrm{H}, \mathrm{CH}_{2}-\mathrm{N}-\mathrm{CH}_{2}\right), 4.91\left(\mathrm{~s}, 2 \mathrm{H},-\mathrm{NH}_{2}\right)$, $6.33(\mathrm{~s}, 1 \mathrm{H}, \mathrm{ArH}), 7.36(\mathrm{t}, J=16 \mathrm{~Hz}, 3 \mathrm{H}, \mathrm{ArH}), 7.53(\mathrm{~d}, J=8 \mathrm{~Hz}, 2 \mathrm{H})$, $7.86(\mathrm{~d}, J=12 \mathrm{~Hz}, 1 \mathrm{H}, \mathrm{ArH}), 8.33(\mathrm{dd}, J=8 \mathrm{~Hz}, 1 \mathrm{H}), 9.98(\mathrm{~d}, J=4 \mathrm{~Hz}$, $1 \mathrm{H})$.

${ }^{13} \mathrm{C}$ NMR (100 MHz, DMSO- $\left.d_{6}\right): \delta=51.2,66.3,100.1,111.7,120.6$, $121.0,121.4,123.5,129.3,129.7,130.6,131.9,136.5,140.2,141.5$, 145.0, 149.4, 158.0, 160.0 .

HRMS (ESI): $m / z$ [MH] $]^{+}$calcd. for $\mathrm{C}_{23} \mathrm{H}_{20} \mathrm{BrN}_{4} \mathrm{O}_{3}$ : 479.0713; found: 481.0689 .

\section{5-(2,4-Dichlorophenyl)-9-nitro-4-(piperidin-1-yl)benzo[ $h] q u i n o-$} lin-2-amine (3m)

Yield: $72 \%$ (168 mg); red solid; $\mathrm{mp} 240-242{ }^{\circ} \mathrm{C}$.

IR (KBr): 3493, $13343 \mathrm{~cm}^{-1}$

${ }^{1} \mathrm{H} \mathrm{NMR}\left(400 \mathrm{MHz}, \mathrm{CDCl}_{3}\right): \delta=0.75-0.82(\mathrm{~m} 2 \mathrm{H}), 1.24-1.26(\mathrm{~m}, 2 \mathrm{H})$, 2.10-2.18 (m, $2 \mathrm{H}), 2.51-2.55(\mathrm{~m}, 2 \mathrm{H}), 2.74(\mathrm{~d}, J=12 \mathrm{~Hz}, 1 \mathrm{H}), 3.03(\mathrm{~d}$, $\left.J=12 \mathrm{~Hz}, 1 \mathrm{H}, \mathrm{CH}_{2}-\mathrm{N}-\mathrm{CH}_{2}\right), 4.81\left(\mathrm{~s}, 2 \mathrm{H},-\mathrm{NH}_{2}\right), 6.37(\mathrm{~s}, 1 \mathrm{H}, \mathrm{ArH}), 7.25-$ $7.30(\mathrm{~m}, 2 \mathrm{H}, \mathrm{ArH}), 7.36-7.37(\mathrm{~m}, 2 \mathrm{H}, \mathrm{ArH}), 7.4(\mathrm{~d}, J=8 \mathrm{~Hz}, 1 \mathrm{H}, \mathrm{ArH})$, $7.78(\mathrm{~d}, J=12 \mathrm{~Hz}, 1 \mathrm{H}, \mathrm{ArH}), 8.26(\mathrm{dd}, J=12 \mathrm{~Hz}, 1 \mathrm{H}, \mathrm{ArH}), 9.96-9.97$ (m, $1 \mathrm{H}, \mathrm{ArH})$.

${ }^{13} \mathrm{C}$ NMR $\left(100 \mathrm{MHz}\right.$, DMSO- $\left.d_{6}\right): \delta=25.0,29.0,55.0,101.2,114.0$, 120.7, 120.8, 121.0, 121.2, 127.4, 127.6, 129.1, 129.3, 132.0, 135.7, $136.8,140.5,145.0,159.5,160.2$.

HRMS (ESI): $m / z$ [MH] $]^{+}$calcd. for $\mathrm{C}_{24} \mathrm{H}_{21} \mathrm{Cl}_{2} \mathrm{~N}_{4} \mathrm{O}_{2}$ : 467.1036; found: 467.1034.

\section{5-(2,4-Dichlorophenyl)-4-morpholino-9-nitrobenzo[ $h]$ quinolin- 2-amine (3n)}

Yield: $74 \%$ (173 mg); red solid; mp $180-182{ }^{\circ} \mathrm{C}$.
IR (KBr): 3356, $1334 \mathrm{~cm}^{-1}$.

${ }^{1} \mathrm{H} \mathrm{NMR}\left(400 \mathrm{MHz}, \mathrm{CDCl}_{3}\right): \delta=2.25-2.34(\mathrm{~m}, 2 \mathrm{H}), 2.47-2.52(\mathrm{~m}, 1 \mathrm{H})$, $2.71(\mathrm{~d}, J=12 \mathrm{~Hz}, 1 \mathrm{H}), 2.90-3.01(\mathrm{~m}, 2 \mathrm{H}), 3.46(\mathrm{~d}, J=12 \mathrm{~Hz}, 1 \mathrm{H}), 3.75$ $(\mathrm{d}, J=8 \mathrm{~Hz}, 1 \mathrm{H}), 5.29\left(\mathrm{~s}, 2 \mathrm{H},-\mathrm{NH}_{2}\right), 6.47(\mathrm{~s}, 1 \mathrm{H}, \mathrm{ArH}), 7.36-7.39(\mathrm{~m}$, $2 \mathrm{H}, \mathrm{ArH}), 7.45(\mathrm{~s}, 1 \mathrm{H}, \mathrm{ArH}), 7.47-7.48(\mathrm{~m}, 1 \mathrm{H}, \mathrm{ArH}), 7.86(\mathrm{~d}, J=8 \mathrm{~Hz}$, $1 \mathrm{H}, \mathrm{ArH}), 8.32(\mathrm{dd}, J=8 \mathrm{~Hz}, 1 \mathrm{H}, \mathrm{ArH}), 10.02-10.03(\mathrm{~m}, 1 \mathrm{H}, \mathrm{ArH})$.

${ }^{13} \mathrm{C}$ NMR $\left(100 \mathrm{MHz}, \mathrm{CDCl}_{3}\right): \delta=53.6,66.0,100.3,114.6,121.5,122.0$, $126.1,126.6,128.6,128.7,131.2,133.6,135.5,136.0,136.3,140.2$, $145.8,158.0,160.1$.

HRMS (ESI): $m / z$ [MH] ${ }^{+}$calcd. for $\mathrm{C}_{23} \mathrm{H}_{19} \mathrm{Cl}_{2} \mathrm{~N}_{4} \mathrm{O}_{3}$ : 469.0829; found: 469.0823.

9-Nitro-4-(piperidin-1-yl)-5-(thiophen-2-yl)benzo[h]quinolin-2amine (3o)

Yield: $80 \%$ (162 mg); red solid; $\mathrm{mp} 130-132{ }^{\circ} \mathrm{C}$.

IR (KBr): $3354,1331 \mathrm{~cm}^{-1}$.

${ }^{1} \mathrm{H}$ NMR $\left(400 \mathrm{MHz}, \mathrm{CDCl}_{3}\right): \delta=1.14-1.23(\mathrm{~m}, 2 \mathrm{H}), 1.37-1.48(\mathrm{~m}, 2 \mathrm{H})$, 2.88-2.93 (m, $2 \mathrm{H}), 3.16-3.22(\mathrm{~m}, 2 \mathrm{H}, \mathrm{ArH}), 4.88\left(\mathrm{~s}, 2 \mathrm{H},-\mathrm{NH}_{2}\right), 7.05-$ $7.07(\mathrm{~m}, 1 \mathrm{H}, \mathrm{ArH}), 7.33-7.36(\mathrm{~m}, 3 \mathrm{H}, \mathrm{ArH}), 7.83(\mathrm{~d}, J=8 \mathrm{~Hz}, \mathrm{ArH})$, 8.21 (dd, $J=8 \mathrm{~Hz}, 1 \mathrm{H}, \mathrm{ArH}), 8.82(\mathrm{~s}, 1 \mathrm{H}, \mathrm{ArH})$.

${ }^{13} \mathrm{C}$ NMR $\left(100 \mathrm{MHz}, \mathrm{CDCl}_{3}\right): \delta=23.6,65.8,119.0,119.2,119.5,120.6$, $120.7,121.3,126.6,127.1,127.3,127.4,130.0,135.5,136.7,140.8$, $143.0,144.6,159.8$

HRMS (ESI): $m / z[M H]^{+}$calcd. for $\mathrm{C}_{22} \mathrm{H}_{21} \mathrm{~N}_{4} \mathrm{O}_{2} \mathrm{~S}$ : 405.1380; found: 405.1375 .

\section{4-Morpholino-9-nitro-5-(thiophen-2-yl)benzo[ $h]$ quinolin-2- amine (3p)}

Yield: $84 \%$ (171 mg); red solid; $\mathrm{mp} 180-182{ }^{\circ} \mathrm{C}$.

IR (KBr): $3380,1372 \mathrm{~cm}^{-1}$.

${ }^{1} \mathrm{H} \mathrm{NMR}\left(400 \mathrm{MHz}, \mathrm{CDCl}_{3}\right): \delta=2.69-2.75(\mathrm{~m}, 2 \mathrm{H}), 2.84-2.90(\mathrm{~m}, 2 \mathrm{H})$, 3.00-3.04 (m, $\left.2 \mathrm{H}, \mathrm{CH}_{2}-\mathrm{N}-\mathrm{CH}_{2}\right), 3.60-3.64\left(\mathrm{~m}, 2 \mathrm{H}, \mathrm{CH}_{2}-\mathrm{N}-\mathrm{CH}_{2}\right), 4.91$ (s, $\left.2 \mathrm{H},-\mathrm{NH}_{2}\right), 6.35(\mathrm{~s}, 1 \mathrm{H}, \mathrm{ArH}), 7.09-7.11(\mathrm{~m}, 1 \mathrm{H}, \mathrm{ArH}), 7.17-7.18$ (m, $1 \mathrm{H}, \mathrm{ArH}), 7.38-7.40(\mathrm{~m}, 1 \mathrm{H}, \operatorname{ArH}), 7.58(\mathrm{~s}, 1 \mathrm{H}, \operatorname{ArH}), 7.85$ (d, $J=$ $8 \mathrm{~Hz}, 1 \mathrm{H}, \mathrm{ArH}), 8.34(\mathrm{dd}, J=8 \mathrm{~Hz}, 1 \mathrm{H}, \mathrm{ArH}), 9.98(\mathrm{~d}, J=4 \mathrm{~Hz}, 1 \mathrm{H}$, $\mathrm{ArH})$.

${ }^{13} \mathrm{C}$ NMR $\left(100 \mathrm{MHz}, \mathrm{CDCl}_{3}\right): \delta=51.6,65.7,98.6,113.1,121.6,122.0$, $125.4,125.6,126.4,127.1,128.4,131.0,133.3,136.2,143.8,145.4$, $145.5,157.7,159.0$

HRMS (ESI): $m / z$ [MH] ${ }^{+}$calcd. for $\mathrm{C}_{21} \mathrm{H}_{19} \mathrm{~N}_{4} \mathrm{O}_{3} \mathrm{~S}$ : 407.1172; found: 407.1169.

4-(4-Benzylpiperazin-1-yl)-5-(4-methoxyphenyl)-9-nitro-4a,10bdihydrobenzo[ $h]$ quinolin-2-amine (3q)

Yield: $75 \%$ (195 mg); red solid; $\mathrm{mp}>250{ }^{\circ} \mathrm{C}$.

IR (KBr): 3356, $1362 \mathrm{~cm}^{-1}$.

${ }^{1} \mathrm{H} \mathrm{NMR}\left(400 \mathrm{MHz}, \mathrm{CDCl}_{3}\right): \delta=2.43(\mathrm{~s}, 4 \mathrm{H}), 3.40\left(\mathrm{~s}, 2 \mathrm{H},-\mathrm{CH}_{2}\right), 3.78(\mathrm{~s}$, $4 \mathrm{H}), 3.85$ (s, $3 \mathrm{H},-\mathrm{OMe}), 4.85\left(\mathrm{~s}, 2 \mathrm{H},-\mathrm{NH}_{2}\right), 6.9(\mathrm{~d}, J=8 \mathrm{~Hz}, 2 \mathrm{H}, \mathrm{ArH})$, $7.27(\mathrm{~s}, 5 \mathrm{H}, \mathrm{ArH}), 7.36(\mathrm{~d}, J=8 \mathrm{~Hz}, 2 \mathrm{H}, \operatorname{ArH}), 7.82(\mathrm{~d}, J=8 \mathrm{~Hz}, 1 \mathrm{H}$, $\mathrm{ArH}), 8.22(\mathrm{dd}, J=8 \mathrm{~Hz}, 1 \mathrm{H}, \mathrm{ArH}), 8.85(\mathrm{~s}, 1 \mathrm{H}, \mathrm{ArH})$.

${ }^{13} \mathrm{C}$ NMR $\left(100 \mathrm{MHz}\right.$, DMSO- $\left.d_{6}\right): \delta=53.0,55.7,62.2,67.0,113.5,113.7$, $116.6,120.1,120.4,121.4,122.1,127.5,128.7,129.3,129.5,130.0$, $130.1,133.0,137.3,143.8,144.1,145.3,159.4,160.7$.

HRMS (ESI): $m / z$ [MH] ${ }^{+}$calcd. for $\mathrm{C}_{31} \mathrm{H}_{32} \mathrm{~N}_{5} \mathrm{O}_{3}$ : 522.2500; found: 522.2502 . 
5-(4-Methoxyphenyl)-4-(4-methylpiperazin-1-yl)-9-nitro-4a,10bdihydrobenzo[ $h]$ quinolin-2-amine (3r)

Yield: $72 \%$ (161 mg); red solid; $\mathrm{mp} 210-212^{\circ} \mathrm{C}$.

IR (KBr): $3361,1381 \mathrm{~cm}^{-1}$.

${ }^{1} \mathrm{H}$ NMR (400 MHz, $\mathrm{CDCl}_{3}$ ): $\delta=1.23(\mathrm{~s}, 2 \mathrm{H}), 1.67$ (s, $\left.3 \mathrm{H}\right), 2.16(\mathrm{~s}, 3 \mathrm{H}$, $\left.\mathrm{N}-\mathrm{CH}_{3}\right), 2.98-3.00(\mathrm{~m}, 3 \mathrm{H}),, 3.83(\mathrm{~s}, 3 \mathrm{H},-\mathrm{OMe}), 4.88\left(\mathrm{~s}, 2 \mathrm{H},-\mathrm{NH}_{2}\right)$, $6.91(\mathrm{~d}, J=8 \mathrm{~Hz}, 2 \mathrm{H}, \mathrm{ArH}), 7.28(\mathrm{~S}, 1 \mathrm{H}, \mathrm{ArH}), 7.35-7.39(\mathrm{~m}, 3 \mathrm{H}, \mathrm{ArH})$, $7.83(\mathrm{~d}, J=8 \mathrm{~Hz}, 1 \mathrm{H}, \mathrm{ArH}), 8.22$ (dd, $J=8 \mathrm{~Hz}, 1 \mathrm{H}, \mathrm{ArH}), 8.85-8.85$ (m, $1 \mathrm{H}, \mathrm{ArH})$.

${ }^{13} \mathrm{C}$ NMR $\left(100 \mathrm{MHz}, \mathrm{CDCl}_{3}\right): \delta=45.7,49.0,52.2,67.7,113.3,113.4$, $114.5,119.1,119.2,120.5,120.7,120.8,129.8,129.9,132.3,137.0$, $142.8,143.0,144.5,159.5,160.4$

HRMS (ESI): $m / z$ [MH] ${ }^{+}$calcd. for $\mathrm{C}_{25} \mathrm{H}_{28} \mathrm{~N}_{5} \mathrm{O}_{3}$ : 446.2187; found: 446.2185.

\section{4-(2-Cyano-4-nitrobenzyl)-2-oxo-6-phenyl-2H-pyran-3-carboni-} trile (5a)

Yield: $71 \%$ (127 mg); red solid; $\mathrm{mp} 220-222{ }^{\circ} \mathrm{C}$.

IR (KBr): 2225, 1733, $1520 \mathrm{~cm}^{-1}$.

${ }^{1} \mathrm{H}$ NMR $\left(400 \mathrm{MHz}, \mathrm{CDCl}_{3}\right): \delta=4.41\left(\mathrm{~s}, 2 \mathrm{H},-\mathrm{CH}_{2}\right), 6.83(\mathrm{~s}, 1 \mathrm{H}, \mathrm{ArH})$, 7.45-7.49 (m, $3 \mathrm{H}, \mathrm{ArH}), 7.75-7.79(\mathrm{~m}, 2 \mathrm{H}, \mathrm{ArH}), 7.82(\mathrm{~d}, J=8 \mathrm{~Hz}, 1 \mathrm{H}$, ArH), 8.46 (dd, $J=8 \mathrm{~Hz}, 1 \mathrm{H}, \mathrm{ArH}), 8.58$ (d, $J=4 \mathrm{~Hz}, 1 \mathrm{H}, \mathrm{ArH}$ ).

${ }^{13} \mathrm{C}$ NMR (100 MHz, DMSO- $\left.d_{6}\right): \delta=29.0,98.7,104.2,114.1,116.0$, 116.4, 117.3, 128.1, 128.4, 129.3, 129.4, 131.3, 145.8, 145.9, 158.1, $161.8,164.1$

HRMS (ESI): $m / z$ [MH] ${ }^{+}$calcd. for $\mathrm{C}_{20} \mathrm{H}_{21} \mathrm{~N}_{3} \mathrm{O}_{4}$ : 358.0822; found: 358.0820 .

\section{4-(2-Cyano-4-nitrobenzyl)-6-(4-methoxyphenyl)-2-oxo-2H- pyran-3-carbonitrile $\mathbf{( 5 b )}$}

Yield: 75\% (134 mg); red solid; $\mathrm{mp} 205-20{ }^{\circ} \mathrm{C}$.

IR (KBr): 2225, 1729, $1504 \mathrm{~cm}^{-1}$.

${ }^{1} \mathrm{H}$ NMR (400 MHz, $\left.\mathrm{CDCl}_{3}\right): \delta=3.98(\mathrm{~s}, 3 \mathrm{H},-\mathrm{OMe}), 4.40\left(\mathrm{~s}, 2 \mathrm{H},-\mathrm{CH}_{2}\right.$ ), $6.75(\mathrm{~s}, 1 \mathrm{H}, \mathrm{ArH}), 6.98-7.00$ (m, $2 \mathrm{H}, \mathrm{ArH}), 7.81-7.85(\mathrm{~m}, 3 \mathrm{H}, \mathrm{ArH})$, $8.47(\mathrm{dd}, J=4 \mathrm{~Hz}, 1 \mathrm{H}, \mathrm{ArH}), 8.59(\mathrm{~d}, J=4 \mathrm{~Hz}, 1 \mathrm{H})$.

${ }^{13} \mathrm{C}$ NMR $\left(100 \mathrm{MHz}\right.$, DMSO- $\left.d_{6}\right): \delta=29.0,55.7,96.6,102.7,113.8$, 114.2, 114.9, 115.8, 121.7, 128.4, 128.7, 131.6, 146.1, 146.8, 158.4, 163.0, 163.2, 164.0.

HRMS (ESI): $m / z$ calcd. for $\mathrm{C}_{21} \mathrm{H}_{14} \mathrm{~N}_{3} \mathrm{O}_{5}$ : 388.0928; found: 388.0927 .

4-(2-Cyano-4-nitrobenzyl)-6-(4-fluorophenyl)-2-oxo-2H-pyran-3carbonitrile (5c)

Yield: $74 \%$ (139 mg); red solid; mp $225-227^{\circ} \mathrm{C}$.

IR (KBr): 2226, 1730, $1528 \mathrm{~cm}^{-1}$.

$\left.{ }^{1} \mathrm{H} \mathrm{NMR} \mathrm{(400} \mathrm{MHz,} \mathrm{CDCl}_{3}\right): \delta=4.42\left(\mathrm{~s}, 2 \mathrm{H},-\mathrm{CH}_{2}\right), 7.15(\mathrm{~s}, 1 \mathrm{H}, \mathrm{ArH})$, $7.35(\mathrm{t}, J=16 \mathrm{~Hz}, 2 \mathrm{H}, \mathrm{ArH}), 7.75(\mathrm{~d}, J=8 \mathrm{~Hz}, 1 \mathrm{H}, \mathrm{ArH}), 7.91-7.95(\mathrm{~m}$, $2 \mathrm{H}, \mathrm{ArH}), 8.42$ (dd, $J=4 \mathrm{~Hz}, 1 \mathrm{H}, \mathrm{ArH}), 8.77$ (d, $J=4 \mathrm{~Hz}, 1 \mathrm{H}, \mathrm{ArH})$.

${ }^{13} \mathrm{C}$ NMR $\left(100 \mathrm{MHz}\right.$, DMSO- $\left.d_{6}\right): \delta=29.0,98.7,104.1,114.1,115.8$, $116.6\left(\mathrm{~d}, J_{\mathrm{C}-\mathrm{F}}=30 \mathrm{~Hz}\right), 128.2\left(\mathrm{~d}, J_{\mathrm{C}-\mathrm{F}}=30 \mathrm{~Hz}\right), 129.4\left(\mathrm{~d}, J_{\mathrm{C}-\mathrm{F}}=20 \mathrm{~Hz}\right)$, 131.8, 146.0, 145.8, 158.1, 161.8, 158.1, 161.

HRMS (ESI): $m / z[\mathrm{MH}]^{+}$calcd. for $\mathrm{C}_{20} \mathrm{H}_{11} \mathrm{FN}_{3} \mathrm{O}_{4}$ : 376.0728; found: 376.0726

6-(4-Chlorophenyl)-4-(2-cyano-4-nitrobenzyl)-2-oxo-2H-pyran3-carbonitrile (5d)

Yield: 74\% (145 mg); red solid; $\mathrm{mp} 215-217^{\circ} \mathrm{C}$.
IR (KBr): 2228, 1729, $1526 \mathrm{~cm}^{-1}$.

${ }^{1} \mathrm{H}$ NMR $\left(400 \mathrm{MHz}, \mathrm{CDCl}_{3}\right): \delta=4.43\left(\mathrm{~s}, 2 \mathrm{H},-\mathrm{CH}_{2}\right), 6.85(\mathrm{~s}, 1 \mathrm{H}, \mathrm{ArH})$, 7.48 (d, $J=8 \mathrm{~Hz}, 3 \mathrm{H}, \mathrm{ArH}), 7.78$ (d, $J=8 \mathrm{~Hz}, 2 \mathrm{H}, \mathrm{ArH}$ ), 8.48 (dd, $J=$ $4 \mathrm{~Hz}, 1 \mathrm{H}, \mathrm{ArH}), 8.60(\mathrm{~d}, J=4 \mathrm{~Hz}, 1 \mathrm{H}, \mathrm{ArH})$.

${ }^{13} \mathrm{C}$ NMR $\left(100 \mathrm{MHz}\right.$, DMSO- $\left.d_{6}\right): \delta=29.5,99.6,105.0,114.3,116.3$, 128.7, 128.8, 129.0, 128.9, 130.0, 132.2, 138.0, 146.4, 147.3, 158.5, $162.0,164.6$.

HRMS (ESI): $m / z$ [MH] ${ }^{+}$calcd. for $\mathrm{C}_{20} \mathrm{H}_{11} \mathrm{ClN}_{3} \mathrm{O}_{4}$ : 392.0433; found: 392.0438 .

3-(1-Amino-7-nitro-3-(thiophen-2-yl)naphthalen-2-yl)-3-(piperidin-1-yl)acrylonitrile (7)

Yield: 80\% (162 mg); red solid; $\mathrm{mp} 170-172{ }^{\circ} \mathrm{C}$.

IR (KBr): 3356, 2214, $1334 \mathrm{~cm}^{-1}$.

${ }^{1} \mathrm{H} \mathrm{NMR}\left(400 \mathrm{MHz}, \mathrm{CDCl}_{3}\right): \delta=1.14-1.23(\mathrm{~m}, 2 \mathrm{H}), 1.37-1.48(\mathrm{~m}, 2 \mathrm{H})$, 2.88-2.93 (m, $\left.2 \mathrm{H}, \mathrm{CH}_{2}-\mathrm{N}-\mathrm{CH}_{2}\right), 3.16-3.22\left(\mathrm{~m}, 2 \mathrm{H}, \mathrm{CH}_{2}-\mathrm{N}-\mathrm{CH}_{2}\right), 4.33$ $(\mathrm{s}, 1 \mathrm{H},=\mathrm{CH}-\mathrm{CN}), 4.88\left(\mathrm{~s}, 2 \mathrm{H},-\mathrm{NH}_{2}\right), 7.05-7.07(\mathrm{~m}, 1 \mathrm{H}, \mathrm{ArH}), 7.33-$ $7.36(\mathrm{~m}, 3 \mathrm{H}, \mathrm{ArH}), 7.48(\mathrm{~s}, 1 \mathrm{H}, \mathrm{ArH}), 7.83(\mathrm{~d}, J=8 \mathrm{~Hz}, 1 \mathrm{H}, \mathrm{ArH}), 8.21$ (dd, $J=8 \mathrm{~Hz}, 1 \mathrm{H}, \mathrm{ArH}), 8.82(\mathrm{~s}, 1 \mathrm{H}, \mathrm{ArH})$.

${ }^{13} \mathrm{C}$ NMR $\left(100 \mathrm{MHz}, \mathrm{CDCl}_{3}\right): \delta=23.6,29.6,65.8,114.5,119.0,119.2$, 120.6, 120.7, 121.3, 126.6, 127.1, 127.3, 127.4, 130.0, 135.5, 136.7, $140.8,143.0,144.6,159.8$.

HRMS (ESI): $m / z$ [MH] $]^{+}$calcd. for $\mathrm{C}_{22} \mathrm{H}_{21} \mathrm{~N}_{4} \mathrm{O}_{2} \mathrm{~S}: 405.1380$; found: 405.1379.

\section{Funding Information}

RP thanks CSIR, New Delhi (No. 02(0286)/16/EMR-II) for research funding. RP and $\mathrm{S}$ thank the University Grants Commission (UGC, New Delhi) and RS thanks CSIR, New Delhi for Senior Research Fellowships. The authors thank the University of Delhi for providing research funding and USIC, Delhi University for providing the instrumentation facility

\section{Supporting Information}

Supporting information for this article is available online at https://doi.org/10.1055/s-0037-1610665.

\section{References}

(1) Tewey, K. M.; Chen, G. L.; Nelson, E. M.; Liu, L. F. J. Biol. Chem. 1984, 259, 9182.

(2) Pommier, Y.; Covey, J. M.; Kerrigan, D.; Markovits, J.; Pharm, R. Nucleic Acids Res. 1987, 15, 6713.

(3) Larsen, A. K.; Grondard, L.; Couprie, J.; Desoize, B.; Comoe, L.; Jardillier, J. C.; Riou, J. F. Biochem. Pharmacol. 1993, 46, 1403.

(4) Makhey, D.; Gatto, B.; Yu, C.; Liu, A.; Liu, L. F.; LaVoie, E. J. Med. Chem. Res. 1996, 56, 2795.

(5) Nabiev, I.; Chourpa, I.; Riou, J. F.; Nguyen, C. H.; Lavelle, F.; Manfait, M. Biochemistry 1994, 33, 9013.

(6) (a) Zishiri, V. K.; Joshi, M. C.; Hunter, R.; Chibale, K.; Smith, P. J.; Summers, R. L.; Martin, R. E.; Egan, T. J. J. Med. Chem. 2011, 54, 6959. (b) Bellot, F.; Coslédéric, F.; Vendier, L.; Brocard, J.; Meunier, B.; Robert, A. J. Med. Chem. 2010, 53, 4103. (c) Klingenstein, R.; Melnyk, P.; Leliveld, S. R.; Ryckebusch, A.; Korth, C. J. Med. Chem. 2006, 49, 5300.

(7) Michael, J. P. Nat. Prod. Rep. 2008, 25, 166. 
(8) Chen, Y. W.; Chen, Y. L.; Tseng, C. H.; Liang, C. C.; Yang, C. N.; Yao, Y. C.; Lu, P. J.; Tzeng, C. C. J. Med. Chem. 2011, 54, 4446.

(9) Lilienkampf, A.; Mao, J.; Wan, B.; Wang, Y.; Franzblau, S. G.; Kozikowski, A. P. J. Med. Chem. 2009, 52, 2109.

(10) (a) Nandhakumar, R.; Suresh, T.; Jude, A. L. C.; Kannan, V. R.; Mohan, P. S. Eur. J. Med. Chem. 2007, 42, 1128. (b) Kategaonkar, A. H.; Shinde, P. V.; Kategaonhar, A. H.; Pasale, S. K.; Shingate, B. B.; Shingare, M. S. Eur. J. Med. Chem. 2010, 45, 3142. (c) Sabatini, S.; Gosetto, F.; Manfroni, G.; Tabarrini, O.; Kaatz, G. W.; Patel, D.; Cecchetti, V.J. Med. Chem. 2011, 54, 5722.

(11) Mahata, P. K.; Venkatesh, C.; Kumar, U. K. S.; Ila, H.; Junjappa, H. J. Org. Chem. 2003, 68, 3966.

(12) Piechowska, J.; Gryko, D. T. J. Org. Chem. 2011, 76, 10220.

(13) Younes, L.; Vincent, H.; Chandrasekaran, Y.; Desce, M. B.; Acher, F. C.; Nicolas, P. J. Org. Chem. 2012, 77, 8294.

(14) Yadav, D. K.; Rai, R.; Kumar, N.; Singh, S.; Misra, S.; Sharma, P.; Shaw, P.; Pérez-Sanchez, H.; Mancera, R. L.; Choi, H. E.; Kim, M. H.; Pratap, R. Scientific Reports 2017, 6, 38128.

(15) (a) Jenekhe, S. A.; Lu, L.; Alam, M. M. Macromolecules 2001, 34, 7315. (b) Aggarwal, A. K.; Jenekhe, S. A. Macromolecules 1991, 24, 6806. (c) Zhang, X.; Shetty, A. S.; Jenekhe, S. A. Macromolecules 1999, 32, 7422.

(16) Skraup, Z. H. Monatsh. Chem. 1881, 2, 139.

(17) Doebner, O.; Miller, W. V. Ber. Dtsch. Chem. Ges. 1884, 17, 1698.

(18) Cohn, B. E.; Gustavson, R. G. J. Am. Chem. Soc. 1928, 50, 2709.

(19) Warren, F. L. J. Chem. Soc. 1936, 1366.

(20) Peters, O.; Friedrichsen, W. Tetrahedron Lett. 1995, 36, 8581.

(21) Riesgo, E. C.; Jin, X.; Thummel, R. P. J. Org. Chem. 1996, 61, 3017.

(22) Luo, Y.; Pan, X.; Wu, J. Org. Lett. 2011, 13, 1150.

(23) (a) Sakai, N.; Tamura, K.; Shimamura, K.; Ikeda, R.; Konakahara, T. Org. Lett. 2012, 14, 836. (b) Huang, H.; Jiang, H.; Chen, K.; Liu, H. J. Org. Chem. 2009, 74, 5476.

(24) Korivi, R. P.; Cheng, C. H. J. Org. Chem. 2006, 71, 7079.

(25) Jiang, B.; Si, Y. G. J. Org. Chem. 2002, 67, 9449.

(26) Zhao, P.; Yan, X.; Yin, H.; Xi, C. Org. Lett. 2014, 16, 1120.

(27) Al-Mutairi, T. M.; Al-Hazimi, H. M.; El-Baih, F. M. J. Saudi. Chem. Soc. 2009, 13, 199.

(28) Janin, Y. L.; Bisagni, E.; Carrez, D. J. Heterocycl. Chem. 1993, 30, 1129.

(29) Pratap, R.; Ram, V. J. Tetrahedron Lett. 2007, 48, 2755.
(30) Singh, S.; Yadav, p.; Sahu, S. N.; Sharone, A.; Kumar, B.; Ram, V. J. Synlett 2014, 25, 2599.

(31) Singh, S.; Panwar, R.; Althagafi; Sharma, V.; Chaudhary, S. Tetrahedron Lett. 2015, 56, 5203.

(32) Singh, S.; Panwar, R.; Yadav, P.; Althagafi, I.; Sahu, S. N.; Pratap, R. RSC Adv. 2015, 5, 18335.

(33) (a) Tominaga, Y.; Ushirogochi, A.; Matsuda, Y. J. Heterocycl. Chem. 1987, 24, 1557. (b) Gompper, R.; Kutter, E.; Töpfl, W. Justus Liebigs Ann. Chem. 1962, 659, 90. (c) Ram, V. J.; Verma, M. Indian J. Chem. 1990, 29B, 624.

(34) (a) Pratap, R.; Kumar, B.; Ram, V. J. Tetrahedron 2006, 34, 8158. (b) Pratap, R.; Kumar, B.; Ram, V. J. Tetrahedron 2006, 62, 8158. (c) Farhanullah, F.; Agarwal, N.; Goel, A.; Ram, V. J. J. Org. Chem. 2003, 68, 2983. (d) Tominaga, Y.; Ushirogouchi, A.; Matsuda, Y.; Kobayashi, G. Chem. Pharm. Bull. 1984, 32, 3384. (e) Ram, V. J.; Verma, M.; Hussaini, F. A.; Shoeb, A. J. Chem. Res., Synop. 1991, 98.

(35) Crystal data for $\mathrm{C}_{24} \mathrm{H}_{23} \mathrm{FN}_{4} \mathrm{O}_{2}$ : A red crystal $(0.220 \times 0.200 \times$ $0.180 \mathrm{~mm}^{3}$ ) was mounted on a capillary tube for indexing and intensity data collection at $298 \mathrm{~K}$ with an Oxford Xcalibur Sapphire3 CCD single-crystal diffractometer (Mo K $\alpha$ radiation, $\lambda=$ $0.71073 \AA$ ). Routine Lorentz and polarization corrections were applied, and an absorption correction was performed using the ABSCALE 3 program [CrysAlis Pro software system, Version 171.34; Oxford Diffraction Ltd., Oxford U. K., 2011]. Patterson methods were used to locate the heavy atoms (SHELXS-86), and the remaining atoms were located from successive Fourier maps (SHELXL-97). All the non-hydrogen atoms were refined anisotropically. All hydrogen atoms were calculated after each cycle of refinement using a Riding model, with $\mathrm{C}-\mathrm{H}=0.93 \AA+$ $\mathrm{U}_{\mathrm{iso}}(\mathrm{H})=1.2 \mathrm{U}_{\mathrm{eq}}(\mathrm{C})$ for aromatic $\mathrm{H}$ atoms, and with $\mathrm{C}-\mathrm{H}=0.97 \AA$ $+\mathrm{U}_{\mathrm{iso}}(\mathrm{H})=1.2 \mathrm{U}_{\mathrm{eq}}(\mathrm{C})$ for methylene $\mathrm{H}$ atoms. Crystal data: CCDC No 1862527 for $\mathrm{C}_{24} \mathrm{H}_{23} \mathrm{FN}_{4} \mathrm{O}_{2} ; M_{r}=416.45$, crystal system: monoclinic; space group C $2 / \mathrm{c} ; a=16.9960(14) \AA, b=8.9323(6)$ $\AA, c=26.3854(19 \AA, \alpha=90, \beta=101.583, \gamma=90 ; V=3924.1(5)$; $Z=8, \rho_{\text {calcd }}=1.410 \mathrm{Mg} / \mathrm{m}^{3} \mathrm{~g} \mathrm{~cm}^{-3} ; \mu=0.099 \mathrm{~mm}^{-1} ; \mathrm{F}(000)=$ 1744.0, R1 $=0.0558(3860)$ and $w R 2=01420(5075)$ for $I>2 \sigma(I)$ and 226 parameters, R1 $=0.0558$ (3860) and $\mathrm{wR} 2=01420$ (5075), gof $=0.904$ for all data. 